\title{
Alternations in the Cardiovascular Autonomic Regulation and Growth Factors in Autism
}

\author{
Ingrid TONHAJZEROVA ${ }^{1,4}$, Igor ONDREJKA ${ }^{2}$, Nikola FERENCOVA ${ }^{1,4}$, Iveta \\ BUJNAKOVA ${ }^{3,1}$, Marian GRENDAR ${ }^{4}$, Lucia BONA OLEXOVA ${ }^{1,4}$, Igor HRTANEK ${ }^{2}$, \\ Zuzana VISNOVCOVA ${ }^{4,1}$
}

${ }^{1}$ Department of Physiology, Comenius University in Bratislava, Jessenius Faculty of Medicine in Martin (JFM CU), Martin, Slovak Republic, ${ }^{2}$ Psychiatric Clinic JFM CU, University Hospital Martin, Martin, Slovak Republic, ${ }^{3}$ Society to Help People with Autism (SPOSA - Turiec), Martin, Slovak Republic, ${ }^{4}$ Biomedical Center Martin JFM CU, Martin, Slovak Republic

Received February 16, 2021

Accepted May 18, 2021

Epub Ahead of Print June 2, 2021

\section{Summary}

Autism spectrum disorder (ASD) represents a serious neurodevelopmental disorder associated with autonomic nervous system dysregulation. The aim was to study complex cardiovascular autonomic regulation using heart rate variability (HRV) and systolic blood pressure variability (SBPV) linear/nonlinear analysis at rest and during orthostasis, and to assess plasma levels of epidermal growth factor (EGF) and vascular endothelial growth factor (VEGF) in autistic children. Twenty-five ASD boys and 25 age and gender-matched children at the age 7-15 years were examined. After venous blood taking, continuous ECG and blood pressure biosignals were recorded at rest and during orthostasis. Evaluated parameters: RR intervals, high- and low-frequency band of HRV spectral analysis (HF-HRV, LF-HRV), symbolic dynamics parameters OV \%, 1V \%, 2LV \%, 2UV \%, low- and high-frequency band of SBPV (LF-SBPV, HF-SBPV), systolic, diastolic, mean blood pressure, EGF, VEGF plasma levels. RR intervals were significantly shortened and the HF-HRV, LF-SBPV, HF-SBPV parameters were significantly lower at rest, the HF-HRV and LF-SBPV remained lower during orthostasis in autistic children compared to controls $(p<0.05)$. EGF plasma levels were significantly lower in ASD compared to controls $(p=0.046)$. No significant differences were found in remaining parameters. Our study revealed tachycardia, cardiovagal underactivity, and blunted sympathetic vasomotor regulation at rest and during orthostasis in autistic children. Additionally, complex heart rate dynamics are similar in autistic children than controls. Furthermore, EGF was reduced in autistic children without significant correlations with any autonomic parameters. We suggest that the abnormal complex cardiovascular reflex control could contribute to understanding the pathway linking autonomic features and autism.

\section{Key words}

Autism • Blood pressure variability • Cardiovascular regulation • Growth factors $\bullet$ Heart rate variability

\section{Corresponding author}

Zuzana Visnovcova, Biomedical Center Martin JFM CU, Mala Hora 4C, Martin, Slovak Republic. E-mail: zuzana.visnovcova@uniba.sk

\section{Introduction}

Autism spectrum disorder (ASD) represents a serious neurodevelopmental disorder characterized by a deficit in social communication and interaction associated with the presence of restricted stereotyped interests. Although the ASD's exact etiology is still unknown, several pathomechanisms are supposed such as neuroendocrine mechanisms (Ostatnikova et al. 2016, 2020), or recently discussed gastrointestinal dysfunction or bidirectional "gut-brain" axis involvement (Babinska et al. 2014, Tomova et al. 2015, 2020, Eshraghi et al. 2020). However, growing evidence has shown the association of ASD with the dysfunction of autonomic nervous system. The polyvagal theory (Porges et al. 2013) proposes

PHYSIOLOGICAL RESEARCH • ISSN 1802-9973 (online) - an open access article under the CC BY-NC-ND 4.0 license (c) 2021 Institute of Physiology of the Czech Academy of Sciences, Prague, Czech Republic Fax +420 241062 164, e-mail: physres@fgu.cas.cz, www.biomed.cas.cz/physiolres 
a neurophysiological circuit that includes the myelinated vagus nerve linked to cranial nerves controlling facial expression and vocalization for the regulation of the social engagement system, which deficits result in maladaptive and behavioral characteristics of autism. It seems that increasing vagal activity is crucial for social/ communication behavior. On the other hand, the sympathetic nervous system is engaged predominantly in a "flight or fight" response, leading to a "threat-like" response during social encounters. Therefore, adaptive social engagement would be associated with increased vagal activity and a decrease in the sympathetic nervous system. Since both parasympathetic and sympathetic nerve outputs also affect cardiac activity, the cardiac parameters can be used as a proxy for the relative activity of the cardiac autonomic control in autism (Ellenbroek and Sengul 2017).

The heart rate variability (HRV), i.e. continuous "beat-to-beat" oscillations of heart rate around its mean values, reflects complex cardiac autonomic regulation indicating a healthy and adaptive organism (McCraty and Shaffer 2015). The short-term HRV is routinely analyzed by a linear method quantifying the magnitude of respiratory linked heart rate fluctuations at the highfrequency band (HF-HRV) reflecting mainly parasympathetic regulation (Electrophysiology 1996). Although the study related to the interaction between brain activity (electroencephalography) and respiration was published already 1970 (Faber et al. 1970), current studies are focused on short-term HRV reflecting respiratory sinus arrhythmia. A recent meta-analysis (Cheng et al. 2020) reported that cardiovagal underactivity indexed by low HRV seems to be a potential biomarker of ASD. Nevertheless, the findings are inconsistent, including reduced or unaltered parasympathetic activity (Bujnakova et al. 2016, Lory et al. 2020) or cardiac-linked parasympathetic dominance in children with ASD (Bricout et al. 2018). However, the interpretation of the sympathetic activity based on HRV short-term analysis is controversial (Goldstein et al. 2011). The low-frequency component of HRV (LF-HRV) was previously considered as a betaadrenergic regulation marker (Pagani et al. 1986), but recent studies reported that LF-HRV is mainly determined by the activity of parasympathetic nervous system (Hayano and Yuda 2019). Several studies have indicated that symbolic dynamics - as a non-linear HRV analysis - is superior to conventional spectral indices due to its sensitivity to sympathetically mediated heart rate fluctuations (Porta et al. 2007, Visnovcova et al. 2014).
Our previous studies firstly showed that symbolic dynamics $0 \mathrm{~V} \%$ index reflects the beta-adrenergic activation during cognitive tests in healthy probands (Visnovcova et al. 2014, Mestanik et al. 2015), or in mental disorders (Tonhajzerova et al. 2010, 2016). Therefore, this index might represent novel noninvasive marker of cardiac sympathetic regulation independent of the other effects, such as myocardial preload and afterload influencing frequently used beta-adrenergic index pre-ejection period (Mestanik et al. 2015, Silva et al. 2017). Recent studies revealed that cardiac-linked sympathetic activity was lower in autistic mouse fetuses (Kasahara et al. 2020), or unaltered in autistic children (Muscatello et al. 2021). Moreover, the symbolic dynamics is a suitable method for the qualitative features in complex dynamics of heart rate modulation independent of its magnitude (Voss et al. 2009). To the best of our knowledge, the symbolic dynamics indices have not yet been evaluated in autism. Additionally, several studies supported the use of spectral analysis of systolic blood pressure variability in low-frequency band (LF-SBPV) as a convenient index of alpha-adrenergic vasomotor control (Pagani et al. 1986, Mestanik et al. 2015). However, very little information is known in autistic children, e.g. recent study revealed unaltered sympathetic activation indexed by LF-SBPV in ASD (Bricout et al. 2018). Thus, this question is still unresolved.

Notably, growth factors such as vascular endothelial growth factor (VEGF) and epidermal growth factor (EGF), play a critical role in the neuronal growth, differentiation, proliferation and regulation of neuronal survival and migration; thus, growth factors dysregulation may contribute to ASD pathology (Xu et al. 2015, Macova et al. 2017). Current research is focused on the interplay between ANS and growth factors as potential pathomechanism leading to some pathological states (Magnon 2015). Therefore, we aimed to study complex cardiovascular autonomic regulation using HRV and SBPV linear analysis, and for the first time by HRV non-linear analysis (symbolic dynamics) at rest and during orthostasis in autistic children. Also, EGF and VEGF plasma levels were investigated for the assessment of any associations with autonomic parameters.

\section{Methods}

\section{Ethics statement}

The study was approved by the Ethics 
Committee of the Jessenius Faculty of Medicine in Martin, Comenius University in Bratislava in accordance with the Declaration of Helsinki (2000) of the World Medical Association. All children and their guardians were carefully informed about the study protocol and informed written consent was obtained.

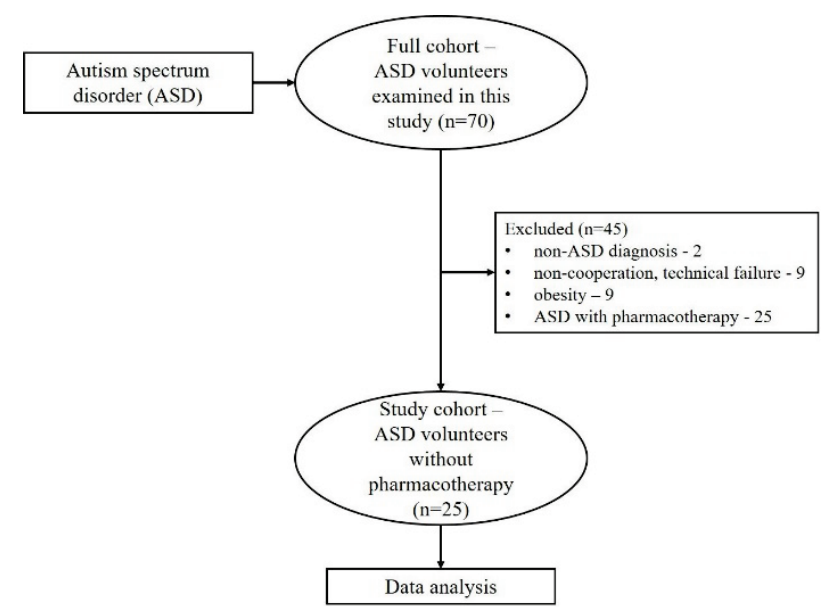

Fig. 1. Cohort flow diagram

\section{Studied groups}

A total of 70 children with ASD diagnosis were examined. According to strict inclusion/exclusion criteria, the final studied group consists of 25 boys suffering from autism spectrum disorder (ASD) at the age between 7-15 years (average age: $11.3 \pm 2.5$ yrs, BMI: $18.6 \pm 3.4 \mathrm{~kg} / \mathrm{m}^{2}$ ) (Fig. 1). The ASD patients were recruited from regional Autism Centre and Psychiatric Clinic University Hospital Martin. The diagnosis was confirmed by a specialist in child and adolescent psychiatry according to the Diagnostic and statistical manual of mental disorder, DSM-5 (American Psychiatric Association 2013). Moreover, the intellectual functioning measured by the Wechsler Intelligence Scale for Children (WISC III) was conducted by a licensed practitioner clinical psychologist. Inclusion criteria for ASD group: ASD diagnosis assessed by a child psychiatrist, IQ above 70, no medication therapy, ability to perform the study protocol. Notably, the ASD group was instructed to avoid potential stress/anxiety effects during the examination.

The control group consisted of 25 age- and gender volunteers (average age: 11.6 \pm 2.6 years, BMI: $18.6 \pm 2.8 \mathrm{~kg} / \mathrm{m}^{2}$ ) without any mental disorders. Exclusion criteria for both groups were following: cardiovascular, endocrine, respiratory diseases, acute respiratory infection, other diseases potentially influencing ANS activity (obesity, underweight, alcohol, drug abuse). Additionally, the body mass index (BMI) of both groups was controlled to age-related percentile graphs within 25th to 75th percentile (Cole and Lobstein 2012) to exclude the potential effect of overweight/obesity or underweight on ANS.

\section{Protocol}

The examination was performed in quiet room under standard conditions (temperature $22-24{ }^{\circ} \mathrm{C}$, air humidity $45-55 \%$, without internal/external disturbances) between 8:00 a.m. to 12:30 p.m., 1 hour after venous blood taking and light breakfast. All examinations were performed in accordance with the ethical principles so that the child did not feel any stress and was as relaxed as possible. The anthropometric parameters - weight, height, body mass index - were examined using direct segmental multi-frequency bioelectrical impedance analysis (InBody 120 Biospace Co., Ltd., Korea). Consequently, the sensors for continuous recording of ECG signal and blood pressure were applied with instructions for posture change to lying position using special armchair. Thereafter, the examination of stress profile was following: rest (lying position) - orthostasis (change of posture from lying to standing). Each experimental period lasted 6 minutes.

\section{Heart rate variability}

The time series of $\mathrm{R}-\mathrm{R}$ intervals were carefully checked for the presence of artifacts, and artifact-free 5 -min sequences were analyzed. Slow fluctuations were filtered using smoothness priors detrending with $\Lambda=500$ (Tarvainen et al. 2002). Consequently, the spectral power in high-frequency band of HRV (HF-HRV, 0.15-0.40Hz), and spectral power in low-frequency band of HRV (LF-HRV, 0.04-0.15Hz) were analyzed using frequencydomain parametric method of autoregressive (AR) spectral estimation using Burg periodogram. For the frequency-domain analyses using Burg periodograms, the time series of RR intervals were resampled using cubic spline interpolation with the frequency $4 \mathrm{~Hz}$ to meet the assumption of a regularly time-sampled signal. Estimation of HF-HRV with Burg periodogram (AR) was performed using model order 16 , which is recommended for the short series of RR intervals with the sampling frequency 2-4 Hz (Boardman et al. 2002). While the HF-HRV is considered as a marker of respiratory-linked heart rate fluctuations mediated through the parasympathetic nervous system, the LF-HRV may be produced by both the sympathetic and parasympathetic nervous system, although resting LF-HRV reflects baroreflex activity and not cardiac sympathetic 
innervation (Shaffer and Ginsberg 2017). Throughout the examination, all children breathed spontaneously with a respiration rate between 14 - 19 breaths per minute. Specifically, the respiratory rate (breaths per minute) was assessed from spectral HRV analysis on the basis of respiratory-related HF frequency (Bailon et al. 2006).

In addition, the RR intervals (ms) were evaluated providing information about the mean heart rate.

\section{Symbolic dynamics}

First, the artefact-free 5-min long sequences of RR intervals were used into transformation in symbolic dynamics analysis. The basic principle of symbolic dynamics algorithm is the transformation of time series (RR intervals) into symbols according to a given alphabet (from 0 to 5). As a result, the symbols were classified into patterns (symbolic words) with length $\mathrm{L}=3$ and were grouped into four families by the types of changes between the symbols. First, patterns without variations (equal to all three symbols) represent an index $0 \mathrm{~V} \%$. Parameter 1V \% corresponds to patterns with one variation (two consecutive equal patterns and the first or third is different). Furthermore, patterns with two different variations form a valley or a peak informing of $2 \mathrm{UV} \%$ and the last index is $2 \mathrm{LV} \%$, which describes two unlike variations form a decreasing or increasing gradient. Symbolic dynamics parameters could reflect changes in sympathovagal balance, meaning that $0 \mathrm{~V} \%$ may represent a potential noninvasive index of betaadrenergic sympathetic control, and $2 \mathrm{UV} \%, 2 \mathrm{LV} \%$ can be cardiovagal indices (Porta et al. 2007, Visnovcova et al. 2014, Mestanik et al. 2015).

\section{Blood pressure variability}

The continuous beat-to-beat blood pressure (BP) signal was continuously noninvasively recorded using Finometer MIDI Model II (Finapres Medical System (FMS), Amsterdam, the Netherlands). Reconstructed brachial artery pressure waveforms were used with the hydrostatic pressure correction of the finger cuff pressure to the heart level via built-in height correction system. The recordings were processed using Beat-Scope Easy software (FMS, Netherlands). First, beat-to-beat series of systolic BP were visually checked to ensure that the segments used for analysis are free of artifacts and the BP series were resampled using cubic spline interpolation with the frequency $2 \mathrm{~Hz}$. Absolute spectral power was calculated in the low-frequency band (LF; $0.075-0.15 \mathrm{~Hz}$ ) and high-frequency band (HF;
0.15-0.40 Hz) of systolic BPV using fast Fourier transform (window width 128 samples, $50 \%$ overlapping). The frequency components LF-SBPV and HF-SBPV reflect predominantly complex sympathetic alpha-adreno-receptor vasomotor and beta-adrenoreceptor cardiac function, respectively (Yoshimoto et al. 2011), although intrinsic vasomotor rhythmicity affected by local mechanisms or respiratory effects may also play a significant role (Stauss 2007).

In addition, the systolic, diastolic and mean blood pressures (SBP, DBP, MBP, respectively) were assessed.

\section{Blood analysis}

Peripheral venous blood was collected into EDTA test-tubes in the morning in fasting state. Then, the blood was centrifuged for 15 minutes at $2500 \mathrm{rpm}$ and $4{ }^{\circ} \mathrm{C}$ (refrigerated centrifugation, Hettich Universal 320R, Tuttlingen, Germany) and the obtained plasma was kept frozen at $-80^{\circ} \mathrm{C}$ until the analysis. Growth factors (EGF, VEGF) were analyzed and quantified using a biochip array technology (Randox, United Kingdom). Biochip Array Technology (BAT) screening on the Evidence Investigator Biochip array technology is a precision multiplex immunoassay-based testing platform allowing for the simultaneous quantitative detection of a wide range of analytes (e.g. growth factors) from a single sample. Plasma assays were provided in a separate kit from Randox Laboratories Ltd. (United Kingdom). Each kit (CTK HS) contains test-biochip cartridges, calibrators, assay diluent, conjugate, wash buffer and signal reagent (luminol and peroxide, 1:1). Quality Controls (CTK HS controls) were used for each run validation. After the addition of $100 \mu \mathrm{l}$ of plasma samples to the biochip, analytes present in the sample bind to the specific biochip bound ligands. The degree of binding is determined using a chemiluminescent light source and quantified using a Charge Coupled Device (CCD) camera and imaging system.

\section{Statistical analysis}

Statistical analysis was performed by SAS University Edition (SAS Institute Inc., Cary, NC, USA). The Shapiro-Wilk test was used for assessment of Gaussian/non-Gaussian distribution. Absolute values of HF-HRV and LF-SBPV differed greatly among individuals, therefore, they were logarithmically transformed for next statistical analysis. Consequently, the two-factor analysis of variance (ANOVA, factors position and group) with repeated measures, and post-hoc

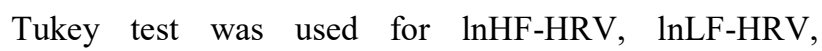


Table 1. HRV and BPV parameters

\begin{tabular}{lccc}
\hline & Control group & ASD group & p-value \\
\hline & Baseline - lying position - HRV parameters & \\
\hline RRinterval $(m s)$ & $837(788-913)$ & $718(666-790)$ & $<0.001$ \\
$\ln L F-H R V\left(\mathrm{~ms}^{2}\right)$ & $1.70 \pm 0.21$ & $1.76 \pm 0.09$ & 0.903 \\
$\ln H F-H R V\left(\mathrm{~ms}^{2}\right)$ & $7.86 \pm 0.85$ & $6.87 \pm 1.44$ & 0.002 \\
OV\% & $27.8(14.9-40.1)$ & $17.8(8.55-37.6)$ & 0.442 \\
$1 V \%$ & $43.3(39.5-46.4)$ & $42.2(35.9-47.6)$ & 0.762 \\
$2 L V \%$ & $7.81(3.53-15.5)$ & $6.7(2.39-11.1)$ & 0.669 \\
$2 U V \%$ & $16.9 \pm 2.37$ & $22.0 \pm 2.80$ & 0.172 \\
respiratory rate (breaths. min $\left.^{-1}\right)$ & $15.80 \pm 0.18$ & $14.85 \pm 0.18$ & 0.309 \\
\hline
\end{tabular}

\begin{tabular}{lccc}
\hline & Baseline - lying position - BPV parameters & \\
\hline$S B P(\mathrm{mmHg})$ & $103.0 \pm 3.03$ & $102.0 \pm 3.21$ & 0.802 \\
$D B P(\mathrm{mmHg})$ & $60.6 \pm 2.50$ & $61.0 \pm 2.25$ & 0.920 \\
$M B P(\mathrm{mmHg})$ & $78.9 \pm 2.58$ & $77.4 \pm 2.51$ & 0.688 \\
$\ln L F-S B P V\left(\mathrm{mmHg}^{2}\right)$ & $6.95 \pm 0.08$ & $5.98 \pm 0.15$ & $<0.001$ \\
$\ln H F-S B P V\left(\mathrm{mmHg}^{2}\right)$ & $6.42 \pm 0.08$ & $5.94 \pm 0.08$ & $<0.001$
\end{tabular}

\begin{tabular}{|c|c|c|c|}
\hline \multicolumn{4}{|c|}{ Active orthostasis - HRV parameters } \\
\hline RRinterval (ms) & $633(608-683)$ & $572(535-618)$ & $<0.001$ \\
\hline $\ln L F-H R V\left(m s^{2}\right)$ & $2.05 \pm 0.23$ & $2.14 \pm 0.09$ & 0.914 \\
\hline $\ln H F-H R V\left(m s^{2}\right)$ & $5.74 \pm 0.98$ & $5.06 \pm 1.36$ & 0.019 \\
\hline$O V \%$ & $31.6(22.6-48.6)$ & $35.4(23.8-56.1)$ & 0.807 \\
\hline $1 \mathrm{~V} \%$ & $45.3(33.8-50.2)$ & $42.7(30.5-47.7)$ & 0.314 \\
\hline $2 L V \%$ & $5.16(1.99-9.02)$ & $4.35(2.20-6.89)$ & 0.714 \\
\hline $2 U V \%$ & $14.2 \pm 1.80$ & $15.2 \pm 1.67$ & 0.999 \\
\hline respiratory rate (breaths. $\mathrm{min}^{-1}$ ) & $18.40 \pm 0.18$ & $18.07 \pm 0.21$ & 0.834 \\
\hline \multicolumn{4}{|c|}{ Active orthostasis - BPV parameters } \\
\hline$S B P(m m H g)$ & $116.0 \pm 4.59$ & $110.0 \pm 3.57$ & 0.843 \\
\hline $\mathrm{DBP}(\mathrm{mmHg})$ & $76.3 \pm 2.60$ & $72.3 \pm 2.50$ & 0.852 \\
\hline$M B P(m m H g)$ & $92.2 \pm 3.06$ & $87.2 \pm 2.72$ & 0.789 \\
\hline $\ln L F-S B P V\left(m m H g^{2}\right)$ & $7.04 \pm 0.06$ & $6.21 \pm 0.14$ & $<0.001$ \\
\hline $\ln H F-S B P V\left(m m H g^{2}\right)$ & $6.25 \pm 0.19$ & $6.24 \pm 0.06$ & 0.968 \\
\hline
\end{tabular}

ASD-autism spectrum disorder; HRV-heart rate variability; BPV-blood pressure variability; InLF-HRV-low-frequency band of HRV; InHF-HRV-high-frequency band of HRV; OV\%, 1V\%, 2LV\%, 2UV\%-indices of symbolic dynamics derived from RR intervals; SBP-systolic blood pressure; DBP-diastolic blood pressure; MBP-mean blood pressure; InLF-SBPV-low-frequency band of systolic BPV (SBPV); InHF-SBPV-high-frequency band of SBPV. Nonparametric data are expressed as median (IQR); parametric data are expressed as mean \pm SEM. $p<0.05$-significant.

Table 2. Growth factors

\begin{tabular}{lccc}
\hline & Control group & ASD group & p-value \\
\hline$E G F(\mathrm{pg} / \mathrm{ml})$ & $19.30(11.00-32.80)$ & $10.50(6.15-25.10)$ & 0.046 \\
$\operatorname{VEGF}(\mathrm{pg} / \mathrm{ml})$ & $9.22(6.11-18.40)$ & $12.10(6.30-17.70)$ & 0.697 \\
\hline
\end{tabular}

EGF-epidermal growth factor; VEGF-vascular endothelial growth factor. Nonparametric data are expressed as median (IQR). $\mathrm{p}<0.05$-significant. 
lnLF-SBPV, lnHF-SBPV, SBP, DBP, MBP, respiratory rate with normal (Gaussian) distribution. The data with non-Gaussian distribution (RR interval, $0 \mathrm{~V} \%, 1 \mathrm{~V} \%$, $2 \mathrm{LV} \%, 2 \mathrm{UV} \%$, EGF, VEGF) were analyzed using the Mann-Whitney test for between-groups comparison. The HRV and SBPV reactivity (\%) to active orthostasis was calculated using formula: (ratio between value during orthostasis and value during baseline phase minus one) $\mathrm{x} 100$. The associations between growth factors (EGF, VEGF) and HRV and between growth factors and SBPV were analyzed by Spearman correlation. Data with Gaussian distribution were expressed as mean $\pm \mathrm{SEM}$, and data with non-Gaussian distribution were expressed as median and interquartile range. The $\mathrm{p}<0.05$ is considered significant.

\section{Results}

The ANOVA revealed significant effect of the group for lnHF-HRV (F[1]=9.08, $p=0.004)$, lnLF-SBPV $(\mathrm{F}[1]=10.42, p=0.003)$ and lnHF-SBPV $(\mathrm{F}[1]=10.08$, $\mathrm{p}=0.004)$. Similarly, the effect of position was significant for lnHF-HRV $(\mathrm{F}[2]=204.86, p<0.001)$ and lnLF-SBPV $(\mathrm{F}[2]=34.51, p<0.001)$. No significant difference was found in the effect of position in parameter lnHF-SBPV. No significant differences were found in the interaction positionxgroup in the indices (lnHF-HRV, lnLF-SBPV and InHF-SBPV). The other parametric distributed parameters (lnLF-HRV, respiratory rate, SBP, DBP, MBP) were without significant effect of the group, effect of the position and in the interaction position $\mathrm{x}$ group.

\section{Post hoc analysis:}

\section{Rest - lying position}

The ASD patients showed significantly decreased parameters InHF-HRV, InHF-SBPV, InLFSBPV compared to controls $(\mathrm{p}=0.002, \mathrm{p}<0.001$ for both lnHF-SBPV, lnLF-SBPV). RR intervals were significantly shortened in ASD group compared to controls $(\mathrm{p}<0.001)$. No significant differences were found in remaining parameters (Table 1 ).

\section{Orthostasis}

The parameters lnHF-HRV, InLF-SBPV were significantly lower in ASD patients compared to controls ( $p=0.019, p<0.001$, respectively). $R R$ intervals remained significantly shortened in ASD patients compared to controls $(p<0.001)$. No significant differences were found in remaining parameters (Table 1). In addition, the HRV and SBPV reactivities were without significant changes between groups.

\section{Growth factors}

EGF was significantly lower in ASD compared to controls $(\mathrm{p}=0.046)$ (Table 2). Moreover, the correlation analysis showed no significant correlations between the growth factors and autonomic parameters.

\section{Discussion}

The main results from this study can be summarized as follows: 1) the short-term HRV linear analysis revealed tachycardia (shortened RR intervals), and decreased cardiovagal control ( $\downarrow$ lnHF-HRV) in autistic children, but HRV symbolic dynamics showed similar complex dynamics of heart rate modulation in autistic children than controls; 2) resting sympatheticallymediated vasomotor regulation was significantly blunted $(\downarrow \operatorname{lnLF-SBPV,} \quad(\downarrow \operatorname{lnHF}-\mathrm{SBPV}) \quad$ in autistic group; 3) abnormal cardiovascular autonomic pattern persisted during orthostasis in ASD; 4) EGF was significantly reduced in ASD.

The autonomic regulation of the effector organs is achieved by multiple structures at all levels of the central nervous system forming a complex interconnected system called central autonomic network (CAN) (Benarroch 1993). Also, the neurovisceral model integrates autonomic, attentional, and affective systems into a functional and structural network (Thayer and Lane 2000). Thus, the understanding of cardiovascular dysregulation in autism is complicated by a large number of cortical, subcortical, and brainstem structures coordinating autonomic function. Specifically, the prefrontal cortex - as the highest level of this hierarchical model with functional connections to subcortical regions - exerts its inhibitory control over sympathoexcitatory circuits. Thus, under threatening situations, the diminished prefrontal inhibitory influence on subcortical regions results in disinhibition of sympathetic circuits associated with cardiovagal underactivity as threat response (Park and Thayer 2014). In this context, our results of cardiac vagal underactivity associated with tachycardia in autistic children confirm the hypothesis of the autonomic hyperarousal indicating a chronic biological threat response in ASD (Patriquin et al. 2019). Hence, we suggest that structural/functional impairment of the prefrontal cortex, such as abnormal cortical cytoarchitecture (Stoner et al. 2014), or altered excitatory 
glutamate/inhibitory GABA systems resulting in impaired functional connectivity (Ajram et al. 2017), is associated with prefrontal cortex underactivity contributing to autonomic dysfunction in autism, as found in our study. Moreover, the neurovisceral integration model proposes that other higher structures contribute to the HF-HRV inhibition including the amygdala, cingulate cortex, and insula, each acting along cortico-inhibitory pathways (Benevides and Lane 2015). The abnormalities of these brain regions have been found in autistic patients (Guo et al. 2017). Furthermore, the polyvagal theory suggests that activation of the vagus nerve (X) promotes the coordination of other cranial nerves (V, VII, IX, XI) included in social engagement system; thus, insufficient vagal nerve output can result in social engagement system deficits in autism (Porges et al. 2013). However, Patriquin et al. (2019) do not suggest dysfunction of cranial nerves, but rather potential differences of the information flowing from the brain to periphery due to distinct neuroception of safety versus threat in autistic patients. Taken together, we assume complex neurophysiological mechanisms leading to cardiovascular dysregulation in autism.

However, the model of chronic biological threat in ASD should be explored with respect to simultaneous measurement of reactivity (Cheng et al. 2020, Skobrtal et al. 2021). ANS is the principal rapidly reacting system that controls blood pressure and heart rate in various physiological stressors, such as orthostasis. The posture change from lying to standing (orthostasis) measures the dynamic baroreflex-mediated changes in the pattern of a vagal withdrawal associated with sympathetic activation. In our study, reduced cardiovagal activity and blunted sympathetic vasomotor regulation remained during active orthostasis in autistic children. Our results are contrary to recent study revealing no significant differences in LF-SBPV to head-up tilt test in autism (Bricout et al. 2018). Although our results showed decreased SBPV in both low- and high-frequency bands indicating potential ASD-linked blunted sympathetic vasomotor control at rest, only LF-SBPV remained significantly decreased during orthostasis in autistic children. It seems that impaired sympathetically-mediated reflex control could reflect mainly alpha-adrenergic vasomotor dysregulation induced by orthostatic test found in our autistic children. However, further research is required concerning the link between sympathetic activity and SBPV components in humans. Taken together, we suggest that abnormal autonomic response to orthostasis may reflect impaired cardiovascular reflex control in autistic children potentially contributing to symptoms of orthostatic intolerance in autism (Goodman 2016). Moreover, it is known that autonomic dysfunction is related to social, emotional and cognitive behaviors in autism, and different autonomic features have been linked to distinct ASD subtypes, such as ASD with anxiety or aggression (Song et al. 2016). It is questionable whether cardiovascular neural dysregulation found in this study may also be associated with ASD characteristics or it reflects pure abnormalities of the complex sympatheticparasympathetic regulatory network leading to impaired response to orthostasis in autism. Further research to elucidate this important question is needed.

Interestingly, the ANS dysfunction is not feature of ASD themselves, but instead attributable to the high levels of stress in autistic subjects (Taylor et al. 2021). In this context, VEGF and EGF are considered as the biomarkers of chronic stress (Wallensten et al. 2016). Our study revealed significantly decreased EGF in autistic children with some studies suggesting that reduced EGF may negatively impact neurodevelopment in ASD children (Galvez-Contreras et al. 2017). Moreover, reduced growth factors due to long-term stress might increase vulnerability to neuronal damage and cognitive impairment (Dahlman et al. 2019). From this view, the „chronic stress-ANS-growth factors“ pathways are still discussed, also from the perspectives of the ANS as a therapeutic target (Magnon 2015). In this study, none of the growth factors significantly correlated with the HRV/SBPV parameters, therefore, in particular EGF seems to provide independent information on ANS activity in potential ASD-linked stress. Identifying all these aspects may allow establishing the clinical use of cardiovascular autonomic and growth factors as biomarkers in ASD.

\section{Limitations of the study}

Our study included a relatively small group of autistic boys, therefore, the findings need to be independently validated in a larger ASD sample with respect to gender. Clinical measures of anxiety or other symptoms by special scales were not evaluated in our ASD children. We suggest that the interaction between psychiatric symptoms and cardiovascular dysregulation could illuminate the pathway linking autonomic dysfunction and autism. Moreover, cardiovascular control was assessed only to orthostasis, thus, the response to other stressors might bring more information about 
autonomic dysregulation in ASD. In this context, the recent development of the ECG biosignal-based deep learning algorithm predicting cardiac arrhythmias or left ventricular systolic dysfunction (Hannun et al. 2019, Attia et al. 2019) could be also attractive for the detection of other noninvasive biomarkers for ASD-linked cardiac complications. Further research in these fields is needed.

\section{Conclusion}

Our study revealed cardiovagal underactivity associated with tachycardia and blunted sympathetic vasomotor regulation at rest and during orthostasis in autistic children. Moreover, qualitative features of complex heart rate dynamics are similar in autistic children providing thus additional information on cardiac autonomic regulatory integrity in autism. Furthermore, EGF was reduced in autistic children without significant correlations with any autonomic parameters. The abnormal cardiovascular reflex functioning could help to elucidate the pathway linking complex autonomic characteristics and autism.

\section{Conflict of Interest}

There is no conflict of interest.

\section{Acknowledgements}

This work was funded by the Slovak Scientific Grant Agency under grants VEGA 1/0030/22; VEGA1/0190/20 and grants of Comenius University UK/63/2021 and $\mathrm{UK} / 80 / 2021$.

\section{References}

AJRAM LA, HORDER J, MENDEZ MA, GALANOPOULOS A, BRENNAN LP, WICHERS RH, ROBERTSON DM, MURPHY CM, ZINKSTOK J, IVIN G, HEASMAN M, MEEK D, TRICKLEBANK MD, BARKER GJ, LYTHGOE DJ, EDDEN RAE, WILLIAMS SC, MURPHY DGM, MCALONAN GM: Shifting brain inhibitory balance and connectivity of the prefrontal cortex of adults with autism spectrum disorder. Transl Psychiatry 7: e1137, 2017. https://doi.org/10.1038/tp.2017.104

ATTIA ZI, KAPA S, YAO X, LOPEZ-JIMENEZ F, MOHAN TL, PELLIKKA PA, CARTER RE, SHAH ND, FRIEDMAN PA, NOSEWORTHY PA: Prospective validation of a deep learning electrocardiogram algorithm for the detection of left ventricular systolic dysfunction. J Cardiovasc Electrophysiol 30: 668-674, 2019. https://doi.org/10.1111/jce.13889

BABINSKA K, BUCOVA M, DURMANOVA V, LAKATOSOVA S, JANOSIKOVA D, BAKOS J, HLAVATA A, OSTATNIKOVA D: Increased plasma levels of the high mobility group box 1 protein (HMGB1) are associated with a higher score of gastrointestinal dysfunction in individuals with autism. Physiol Res 63: 613-618, 2014. https://doi.org/10.33549/physiolres.932932

BAILON R, SORNMO L, LAGUNA P: A robust method for ECG-based estimation of the respiratory frequency during stress testing. IEEE Trans Biomed Eng 53: 1273-1285, 2006. https://doi.org/10.1109/TBME.2006.871888

BENARROCH EE: The Central autonomic network: functional organization, dysfunction, and perspective. Mayo Clin Proc 68: 988-1001, 1993. https://doi.org/10.1016/S0025-6196(12)62272-1

BENEVIDES TW, LANE SJ: A review of cardiac autonomic measures: considerations for examination of physiological response in children with autism spectrum disorder. J Autism Dev Disord 45: 560-575, 2015. https://doi.org/10.1007/s10803-013-1971-z

BOARDMAN A, SCHLINDWEIN FS, ROCHA AP, LEITE A: A study on the optimum order of autoregressive models for heart rate variability. Physiol Meas 23: 325-336, 2002. https://doi.org/10.1088/0967-3334/23/2/308

BRICOUT VA, PACE M, DUMORTIER L, FAVRE-JUVIN A, GUINOT M: Autonomic responses to head-up tilt test in children with autism spectrum disorders. J Abnorm Child Psychol 46: 1121-1128, 2018. https://doi.org/10.1007/s10802-017-0339-9

BUJNAKOVA I, ONDREJKA I, MESTANIK M, VISNOVCOVA Z, MESTANIKOVA A, HRTANEK I, FLESKOVA D, CALKOVSKA A, TONHAJZEROVA I: Autism spectrum disorder is associated with autonomic underarousal. Physiol Res 65: 673-682, 2016. https://doi.org/10.33549/physiolres.933528

CHENG YC, HUANG YC, HUANG WL: Heart rate variability in individuals with autism spectrum disorders: A metaanalysis. Neurosci Biobehav Rev 118: 463-471, 2020. https://doi.org/10.1016/j.neubiorev.2020.08.007 
COLE TJ, LOBSTEIN T: Extended international (IOTF) body mass index cut-offs for thinness, overweight and obesity. Pediatr Obes 7: 284-294, 2012. https://doi.org/10.1111/j.2047-6310.2012.00064.x

DAHLMAN AS, BLENNOW K, ZETTERBERG H, GLISE K, JONSDOTTIR IH: Growth factors and neurotrophins in patients with stress-related exhaustion disorder. Psychoneuroendocrinology 109: 104415, 2019. https://doi.org/10.1016/j.psyneuen.2019.104415

ELECTROPHYSIOLOGY T F OF THE E S: Heart Rate Variability. Circulation 93: 1043-1065, 1996. https://doi.org/10.1161/01.CIR.93.5.1043

ELLENBROEK B, SENGUL H: Autism spectrum disorders: Autonomic alterations with a special focus on the heart. Hear Mind 1: 78, 2017. https://doi.org/10.4103/hm.hm 5 _ 17

ESHRAGHI RS, DAVIES C, IYENGAR R, PEREZ L, MITTAL R, ESHRAGHI AA: Gut-induced inflammation during development may compromise the blood-brain barrier and predispose to autism spectrum disorder. J Clin Med 10: 27, 2020. https://doi.org/10.3390/jcm10010027

FABER J, TUHACEK M, MESTAN J: Synchronization of EEG activity and respiration. Cesk Neurol 33: 296-301, 1970.

GALVEZ-CONTRERAS AY, CAMPOS-ORDONEZ T, GONZALEZ-CASTANEDA RE, GONZALEZ-PEREZ O: Alterations of growth factors in autism and attention-deficit/hyperactivity disorder. Front Psychiatry 8: 126, 2017. https://doi.org/10.3389/fpsyt.2017.00126

GOLDSTEIN DS, BENTHO O, PARK MY, SHARABI Y: Low-frequency power of heart rate variability is not a measure of cardiac sympathetic tone but may be a measure of modulation of cardiac autonomic outflows by baroreflexes. Exp Physiol 96: 1255-1261, 2011. https://doi.org/10.1113/expphysiol.2010.056259

GOODMAN B: Autonomic dysfunction in autism spectrum disorders (ASD) (P5.117). Neurology 86, 2016.

GUO X, DOMINICK KC, MINAI AA, LI H, ERICKSON CA, LU LJ: Diagnosing autism spectrum disorder from brain resting-state functional connectivity patterns using a deep neural network with a novel feature selection method. Front Neurosci 11: 460, 2017. https://doi.org/10.3389/fnins.2017.00460

HANNUN AY, RAJPURKAR P, HAGHPANAHI M, TISON GH, BOURN C, TUZRAKHIA MP, NG AY: Cardiologist-level arrhythmia detection and classification in ambulatory electrocardiograms using a deep neural network. Nat Med 25: 65-69, 2019. https://doi.org/10.1038/s41591-018-0268-3

HAYANO J, YUDA E: Pitfalls of assessment of autonomic function by heart rate variability. J Physiol Anthropol 38, 2019. https://doi.org/10.1186/s40101-019-0193-2

KASAHARA Y, YOSHIDA C, NAKANISHI K, FUKASE M, SUZUKI A, KIMURA Y: Alterations in the autonomic nerve activities of prenatal autism model mice treated with valproic acid at different developmental stages. Sci Rep 10: 1-13, 2020. https://doi.org/10.1038/s41598-020-74662-0

LORY C, KADLASKAR G, MCNALLY KEEHN R, FRANCIS AL, KEEHN B: Brief report: Reduced heart rate variability in children with autism spectrum disorder. J Autism Dev Disord 50: 4183-4190, 2020. https://doi.org/10.1007/s10803-020-04458-8

MACOVA L, BICIKOVA M, OSTATNIKOVA D, HILL M, STARKA L: Vitamin D, neurosteroids and autism. Physiol Res 66: 333-340, 2017. https://doi.org/10.33549/physiolres.933721

MAGNON C: Role of the autonomic nervous system in tumorigenesis and metastasis. Mol Cell Oncol 2: e975643, 2015. https://doi.org/10.4161/23723556.2014.975643

MCCRATY R, SHAFFER F: Heart rate variability: New perspectives on physiological mechanisms, assessment of selfregulatory capacity, and health risk. Glob Adv Heal Med 4: 46-61, 2015. https://doi.org/10.7453/gahmj.2014.073

MESTANIK M, MESTANIKOVA A, VISNOVCOVA Z, CALKOVSKA A, TONHAJZEROVA I: Cardiovascular sympathetic arousal in response to different mental stressors. Physiol Res 64: 585-594, 2015. https://doi.org/10.33549/physiolres.933217

MUSCATELLO RA, VANDEKAR SN, CORBETT BA: Evidence for decreased parasympathetic response to a novel peer interaction in older children with autism spectrum disorder: a case-control study. J Neurodev Disord 13, 2021. https://doi.org/10.1186/s11689-020-09354-x

OSTATNIKOVA D, KUBRANSKA A, MARCINCAKOVA V, PIVOVARCIOVA A, BABKOVA-DURDIAKOVA J: Neuroendocrine contribution to autism etiology. Act Nerv Super Rediviva 58: 65-68, 2016. 
OSTATNIKOVA D, LAKATOSOVA S, BABKOVA J, HODOSY J, CELEC P: Testosterone and the brain: from cognition to autism. Physiol Res 69: 403-419, 2020. https://doi.org/10.33549/10.33549/physiolres.934592

PAGANI M, LOMBARDI F, GUZZETTI S, RIMOLDI O, FURLAN R, PIZZINELLI P, SANDRONE G, MALFATTO G, DELl'ORTO S, PICCALUGA E: Power spectral analysis of heart rate and arterial pressure variabilities as a marker of sympatho-vagal interaction in man and conscious dog. Circ Res 59: 178-193, 1986. https://doi.org/10.1161/01.RES.59.2.178

PARK G, THAYER JF: From the heart to the mind: cardiac vagal tone modulates top-down and bottom-up visual perception and attention to emotional stimuli. Front Psychol 5: $278,2014$. https://doi.org/10.3389/fpsyg.2014.00278

PATRIQUIN MA, HARTWIG EM, FRIEDMAN BH, PORGES SW, SCARPA A: Autonomic response in autism spectrum disorder: Relationship to social and cognitive functioning. Biol Psychol 145: 185-197, 2019. https://doi.org/10.1016/j.biopsycho.2019.05.004

PORGES SW, MACELLAIO M, STANFILL SD, MCCUE K, LEWIS GF, HARDEN ER, HANDELMAN M, DENVER J, BAZHENOVA OV, HEILMAN KJ: Respiratory sinus arrhythmia and auditory processing in autism: Modifiable deficits of an integrated social engagement system? Int J Psychophysiol 88: 261-270, 2013. https://doi.org/10.1016/j.ijpsycho.2012.11.009

PORTA A, TOBALDINI E, GUZZETTI S, FURLAN R, MONTANO N, GNECCHI-RUSCONE T: Assessment of cardiac autonomic modulation during graded head-up tilt by symbolic analysis of heart rate variability. Am $\mathrm{J}$ Physiol Hear Circ Physiol 293: 702-708, 2007. https://doi.org/10.1152/ajpheart.00006.2007

SHAFFER F, GINSBERG JP. An overview of heart rate variability metrics and norms. Front Public Health 5: 258, 2017.

SILVA LEV, GERALDINI VR, DE OLIVEIRA BP, SILVA CAA, PORTA A, FAZAN R: Comparison between spectral analysis and symbolic dynamics for heart rate variability analysis in the rat. Sci Rep 7, 2017. https://doi.org/10.1038/s41598-017-08888-w

SKOBRTAL P, BAZINKOVA E, CHALUPOVA M: The possibilities of using a stress profile in psychotherapy. Cognitive Remediation Journal 10: 11-13, 2021.

SONG R, LIU J, KONG X: Autonomic dysfunction and autism: subtypes and clinical perspectives. N A J Med Sci 9: 172-180, 2016. https://doi.org/10.5507/crj.2021.002

STAUSS HM: Idetification of blood pressure control mechanisms by power spectral analysis. Clin Exp Pharmacol Physiol 34: 362-368, 2007. https://doi.org/10.1111/j.1440-1681.2007.04588.x

STONER R, CHOW ML, BOYLE MP, SUNKIN SM, MOUTON PR, ROY S, WYNSHAW-BORIS A, COLAMARINO SA, LEIN ES, COURCHESNE E: Patches of disorganization in the neocortex of children with autism. N Engl J Med 370: 1209-1219, 2014. https://doi.org/10.1056/NEJMoa1307491

TARVAINEN MP, RANTA-AHO PO, KARJALAINEN PA: An advanced detrending method with application to HRV analysis. IEEE Trans Biomed Eng 49: 172-175, 2002. https://doi.org/10.1109/10.979357

TAYLOR EC, LIVINGSTON LA, CALLAN MJ, ASHWIN C, SHAH P: Autonomic dysfunction in autism: The roles of anxiety, depression, and stress. Autism 25:744-752, 2021. https://doi.org/10.1177/1362361320985658

THAYER JF, LANE RD: A model of neurovisceral integration in emotion regulation and dysregulation. J Affect Disord 61: 201-216, 2000. https://doi.org/10.1016/S0165-0327(00)00338-4

TOMOVA A, HUSAROVA V, LAKATOSOVA S, BAKOS J, VLKOVA B, BABINSKA K, OSTATNIKOVA D: Gastrointestinal microbiota in children with autism in Slovakia. Physiol Behav 138: 179-187, 2015. https://doi.org/10.1016/i.physbeh.2014.10.033

TOMOVA A, SOLTYS K, REPISKA G, PALKOVA L, FILCIKOVA D, MINARIK G, TURNA J, PROCHOTSKA K, BABINSKA K, OSTATNIKOVA D: Specificity of gut microbiota in children with autism spectrum disorder in Slovakia and its correlation with astrocytes activity marker and specific behavioural patterns. Physiol Behav 214, 2020. https://doi.org/10.1016/j.physbeh.2019.112745

TONHAJZEROVA I, FARSKY I, MESTANIK M, VISNOVCOVA Z, MESTANIKOVA A, HRTANEK I, ONDREJKA I: Symbolic dynamics of heart rate variability - A promising tool to investigate cardiac sympathovagal control in attention deficit/hyperactivity disorder (ADHD)? Can J Physiol Pharmacol 94: 579-587, 2016. https://doi.org/10.1139/cjpp-2015-0375 
TONHAJZEROVA I, ONDREJKA I, JAVORKA K, TURIANIKOVA Z, FARSKY I, JAVORKA M: Cardiac autonomic regulation is impaired in girls with major depression. Prog Neuropsychopharmacol Biol Psychiatry 34: 613-618, 2010. https://doi.org/10.1016/i.pnpbp.2010.02.023

VISNOVCOVA Z, MESTANIK M, JAVORKA M, MOKRA D, GALA M, JURKO A, CALKOVSKA A, TONHAJZEROVA I: Complexity and time asymmetry of heart rate variability are altered in acute mental stress. Physiol Meas 35: 1319-1334, 2014. https://doi.org/10.1088/0967-3334/35/7/1319

VOSS BA, SCHULZ S, SCHROEDER R, BAUMERT M, CAMINAL P: Methods derived from nonlinear dynamics for analysing heart rate variability. Philos Trans R Soc A Math Phys Eng Sci 367: 277-296, 2009. https://doi.org/10.1098/rsta.2008.0232

WALLENSTEN J, ÅSBERG M, NYGREN Å, SZULKIN R, WALLÉN H, MOBARREZ F, NAGER A: Possible biomarkers of chronic stress induced exhaustion - A longitudinal study. PLoS One 11: e0153924, 2016. https://doi.org/10.1371/journal.pone.0153924

XU N, LI X, ZHONG Y: Inflammatory cytokines: Potential biomarkers of immunologic dysfunction in autism spectrum disorders. Mediators Inflamm 2015: 531518, 2015. https://doi.org/10.1155/2015/531518

YOSHIMOTO T, EGUCHI K, SAKURAI H, ODMICHI Y, HASHIMOTO T, ODMICHI M, MORIMOTO A, YAMAGUCHI Y, USHIDA T, IWASE S, SUGENOYA J, KUMAZAWA T: Frequency components of systolic blood pressure variability reflect vasomotor and cardiac sympathetic functions in conscious rats. J Physiol Sci 61: 373-383, 2011. https://doi.org/10.1007/s12576-011-0158-7 